\title{
Diseño de interfaz gráfica basada en Big Data para cálculo de sistemas de tubería de Clase III
}

\section{Design of graphical inteface based in Big Data for calculus of systems of pipelines Class III}

RAZÓN-GONZÁLEZ, Juan Pablo†*, GALLARDO-ALVAREZ, Dennise Ivonne, GARCÍAGUZMÁN, José Miguel y DURÁN-BELMAN, Israel

Instituto Tecnológico Superior de Irapuato, Carretera Irapuato-Silao km 12.5, Col. El Copal, C. P. 36821, Irapuato, Gto

ID $1^{\text {er }}$ Autor: Juan Pablo, Razón-González /ORC ID: 0000-0002-9457-5029, Researcher ID Thomson: S-4919-2018, CVUCONACYT ID: 216902

ID $1^{\text {er }}$ Coautor: Dennise Ivonne, Gallardo-Alvarez / ORC ID: 0000-0002-9197-6425, Researcher ID Thomson: S-49212018, CVU CONACYT ID: 264516

ID $2^{\text {do }}$ Coautor: José Miguel, García-Guzmán / ORC ID: 0000-0001-6505-135X, Researcher ID Thomson: S-5892-2018, CVU CONACYT ID: 470152

ID $3^{\text {er }}$ Coautor: Israel Durán-Belman / ORC ID: 0000-0002-1394-0486, CVU CONACYT ID: 691483

DOI: $10.35429 / J C E .2019 .8 .3 .20 .27$

Recibido 03 de Marzo, 2019; Aceptado 30 Junio, 2019

\begin{abstract}
Resumen
Las necesidades actuales de la industria presentan retos que deben enfrentarse cotidianamente en los diferentes sectores productivos. El uso de las tecnologías de vanguardia y el manejo eficaz de grandes volúmenes de información permiten el desarrollo de nuevas herramientas computacionales que mejoren la precisión y optimicen los tiempos de cálculo en el diseño de tuberías. En este trabajo se presenta la aplicación del Programa de Análisis de Sistemas de Tuberías (PAST), mismo que se desarrolló como una interfaz gráfica en el lenguaje de programación MATLAB ${ }^{\circledR}$; esta aplicación computacional determina el tamaño de la tubería comercial basado en la diferencia de presión, elevación, temperatura del agua y flujo volumétrico como los parámetros críticos de diseño. El algoritmo de solución emplea técnicas de Big Data para la manipulación precisa de grandes volúmenes de información, obteniendo resultados con exactitud en tiempos reducidos sin comprometer la confiabilidad de los sistemas de tuberías; de esta manera se optimiza el proceso de diseño comparado con los métodos analíticos tradicionales.
\end{abstract}

Sistemas de tuberías, Big Data, PAST

\begin{abstract}
Absctract
The current requirements of the industry present challenges that must faced daily in the different productive sectors. The use of the cutting-edge technology and the efficient handling of large volumes of information allow the development of new computational tools that improve accuracy and optimize calculation's times in pipe design. In this work the application is presented of the Piping Systems Analysis Program (PSAP), same that was developed as a graphical interface in the computer language MATLAB®; this computational application determines the size of the commercial pipe based on the pressure difference, elevation, water temperature and volume flow as critical design parameters. The algorithm of solution uses skills of Big Data for the precise handling of large volumes of information, getting accurate results at reduced times without compromising the reliability of pipe systems; this way optimizes the design process compared to traditional analytical methods.
\end{abstract}

Citación: RAZÓN-GONZÁLEZ, Juan Pablo, GALLARDO-ALVAREZ, Dennise Ivonne, GARCÍA-GUZMÁN, José Miguel y DURÁN-BELMAN, Israel. Diseño de interfaz gráfica basada en Big Data para cálculo de sistemas de tubería de Clase III. Revista de Ingeniería Civil. 2019. 3-8: 20-27

\footnotetext{
* Correspondencia del Autor (jurazon@itesi.edu.mx)

$\uparrow$ Investigador contribuyendo como primer autor.
} 


\section{Introducción}

En la actualidad existen diversas aplicaciones de los sistemas de tuberías en ámbitos tan diferentes que van desde el sector agroindustrial primario hasta los procesos productivos más avanzados, estos involucran tanto diferentes tipos de fluidos, así como diversos materiales de las tuberías que se definen de acuerdo a los rangos de operación definidos desde el diseño de cada proceso.

Los tiempos en la industria exigen soluciones que presenten la combinación entre precisión, confiabilidad y rapidez para la implementación de nuevos sistemas que solventen las necesidades de diseño e implementación de los nuevos sistemas de tubería en los diferentes sectores.

Dados los tiempos de respuesta requeridos por el sector productivo actual, resulta poco factible el realizar estos cálculos de forma tradicional y normalmente las áreas de diseño y los departamentos de ingeniería optan por aplicaciones computacionales que permitan reducir el tiempo invertido para este fin $\mathrm{e}$ incrementar la confiabilidad de los resultados.

Comercialmente existen diversas alternativas que ofrecen soluciones a los requerimientos de los sectores productivos a un costo relativamente elevado. Estos programas de cómputo permiten caracterizar ni parametrizar una aproximación real al comportamiento de los fluidos, a las pérdidas energéticas, a las caídas de presión y a los parámetros de diseño de la tubería comercial adecuada para cada aplicación.

En los últimos 70 años los softwares más comunes para cálculo de tubería son el WaterCAD y el WaterGEMS (Bentley, 2018), también el Epanet (EPA, 2017). Estos programas son los que tienen el mayor reconocimiento, pero presentan diferentes limitaciones: no consideran los efectos de las pérdidas energéticas por los accesorios de la tubería y las válvulas; tampoco involucran la fricción de materiales al considerar las tuberías de un material hidráulicamente liso, alejándose de la caracterización apegada a la realidad del sistema que se pretende diseñar.
A partir de estas limitaciones que presentan los softwares comerciales, se desarrolló una aplicación computacional basada en el lenguaje de Matlab® (CERVANTES \& BÁEZ, 2012); misma que se presenta como una interface gráfica capaz de determinar el tamaño de la tubería en función de sus requerimientos de diseño y considerando los efectos del factor de rugosidad de cada material de acuerdo a las ecuaciones de Darcy, Hagen-Poiseuille, Moody (Mataix, 2009) y el nomograma de HazenWilliams (Mott, 2006) también se incluyen las ecuaciones Chèzy, Manning y Darcy-Weisbach para la evaluación de las pérdidas de carga (Jiménez-Medina, 2015).

Esta interface gráfica permite calcular el tamaño de tubería comercial adecuado para las características de operación de un sistema propuesto. Esta aplicación permite seleccionar el sistema de unidades, los accesorios, el tipo de salida del fluido desde el tanque a la red de tubería, el caudal y la rugosidad de la tubería que está definida para cada material; en los casos de tuberías no listadas en el catálogo se puede ingresar el parámetro de rugosidad de forma manual.

Este trabajo presenta el diseño de una aplicación que emplea técnicas de Big Data para el manejo de grandes conjuntos de datos complejos procedentes de nuevas fuentes. Estos conjuntos de datos son tan voluminosos que un software de procesamiento de datos convencional sencillamente no puede gestionarlos. Sin embargo, estos volúmenes masivos de datos pueden utilizarse para abordar problemas empresariales e ingenieriles que antes no hubiera sido posible solucionar (Oracle, 2013).

Para entender qué significa realmente "Big Data", resulta útil conocer ciertos antecedentes históricos, como la definición de Gartner que lo describe como "datos que contienen una mayor variedad y que se presentan en volúmenes crecientes y a una velocidad superior". Esto se conoce como "las tres V": Volumen, Velocidad y Variedad; parámetros a los que se han agregado otras dos "V": Valor y Veracidad (Sicular, 2013). El Volumen en big data hace referencia a la cantidad de datos no estructurados de baja densidad que se procesarán. La Velocidad es el ritmo al que se reciben los datos y al que se le aplica alguna acción. La Variedad hace referencia a los diversos tipos de datos disponibles.

RAZÓN-GONZÁLEZ, Juan Pablo, GALLARDO-ALVAREZ, Dennise Ivonne, GARCÍA-GUZMÁN, José Miguel y DURÁN-BELMAN, Israel. Diseño de interfaz gráfica basada en Big Data para cálculo de sistemas de tubería de Clase III. Revista de Ingeniería Civil. 2019 
Los dos criterios restantes, Valor y Veracidad, agregan un valor intrínseco, sin embargo, no tienen ninguna utilidad hasta que dicho valor se descubre. Resulta igualmente importante determinar la veracidad de los datos y cuánto se puede confiar en ellos (Oracle, 2013).

\section{Materiales y métodos}

Para el análisis de sistemas de tuberías de clase III se presentan diferentes esquemas. El más común se define a través de un par de puntos A y B que representan la entrada y salida del sistema. El primer caso es cuando no hay cambios en la elevación, como se muestra en la figura 1. Esto en la aplicación se ingresa como 0 en los puntos 1 y 2 .

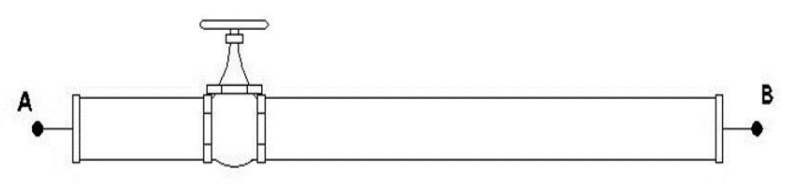

Figura 2 Sistema sin cambio de elevación Fuente: Elaboración Propia

Por otra parte, se tiene el caso donde no hay cambios en la presión ya que los dos tanques se encuentran abiertos a la atmósfera y se consideran con una presión manométrica de 0 , como se presenta en la figura 2.

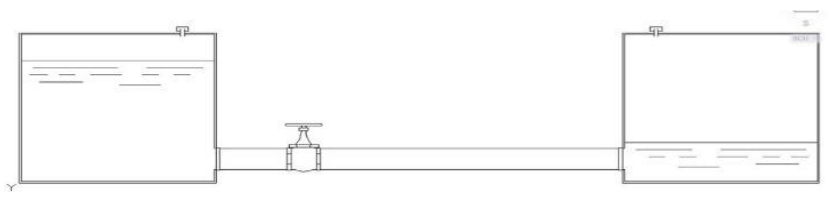

Figura 2 Sistema sin cambios de presión Fuente: Elaboración Propia

En otro caso se puede plantear la caída máxima de presión y expresarlo como una diferencia de presiones entre los puntos 1 y 2 ; esto para que se cumpla con una diferencia de presión en caso de ser requerido. La aplicación permite elegir el sistema de unidades deseado para calcular la tubería de acuerdo a los parámetros de diseño. Al seleccionar el sistema de unidades todos los valores de las tablas se ajustarán a las unidades correspondientes previo a iniciar la simulación. La primera ventana del menú contextual para la elección del sistema de unidades se presenta en la figura 3.

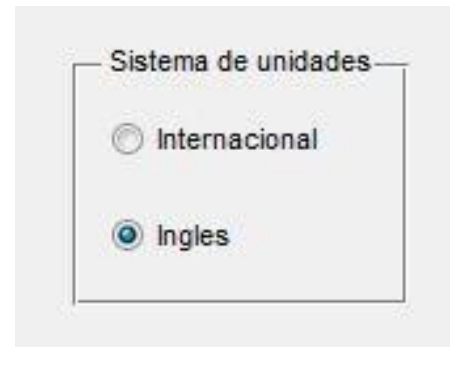

Figura 3 Sistemas de unidades Fuente: Elaboración Propia

La aplicación requiere parámetros que deben ser ingresados para que el algoritmo de solución permita entregar resultados, estos datos de entrada son la longitud de la tubería, diferencia de presión, cambio en las elevaciones y temperatura. De acuerdo al sistema de unidades que se elija, la aplicación le indica al usuario las unidades que debe ingresar para mantener la congruencia durante el proceso de cálculo, tal como se muestra en la figura 4.

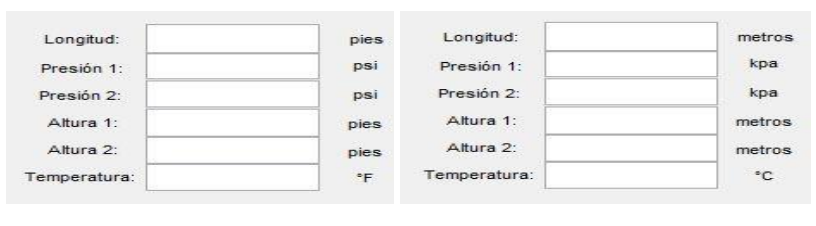

Figura 4 Sistema inglés y sistema internacional de unidades

Fuente: Elaboración Propia

Para la selección del material se cuenta con un catálogo de tuberías comerciales entre los que se pueden elegir los más comunes en el mercado actual: vidrio (Vidrasa, 2017), plástico (Charter-plastics, 2018; PPI, 2018), cobre (Copper-Alliance, 2018; Nacobre, 2016; Herco, 2018), hierro (Dupra, 2015), acero (TubeNet, 2018), entre otros; esto se desplegará a través de un menú; tal como se presenta en la figura 5.

\begin{tabular}{|l|}
\hline Materiales de tubería \\
\hline Materiales de tubería \\
Vidrio \\
Plastico \\
Tubo estruido; cobre \\
Tubo estruido; latón \\
Tubo estruido; acero \\
\hline Acero, comercial o soldado \\
\hline Hierro galvanizado \\
Hierro ductil, recubierto \\
Hierro ductil, no recubierto \\
Concreto, bien fabricado \\
Acero remachado \\
Otro... \\
\hline
\end{tabular}

Figura 5 Lista de materiales de tubería disponibles en la aplicación

Fuente: Elaboración Propia 
Para el caso del acero comercial o soldado, al elegir esta opción se desplegará una ventana que ofrece entre dos opciones: cédula 40 y cédula 80, como se puede ver en la figura 6 .

\begin{tabular}{|l|}
\hline Tipo de cedula \\
\hline Tipo de cedula \\
\hline Cedula 40 \\
Cedula 80 \\
\hline
\end{tabular}

Figura 6 Ventana emergente para seleccionar el tipo de cédula

Fuente: Elaboración Propia

\section{Accesorios}

Este programa cuenta con una serie de accesorios como son los codos y las válvulas, esto con el fin de aproximar a condiciones reales el análisis del sistema hidráulico, incrementando así la precisión. En el menú de la aplicación se presenta un botón deshabilitado para los accesorios, este se activa a través de un "radio button", ya que son diferentes métodos de solución al considerar un análisis con o sin accesorios, de esta manera se indica al algoritmo el método de solución que será empleado; tal como se muestra en la figura 7.

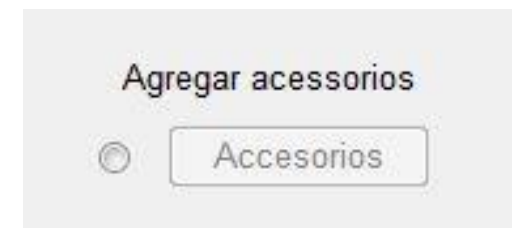

Figura 7 Radio button para selección de método de solución con o sin accesorios

Fuente: Elaboración Propia

Cabe mencionar que en esta versión están disponibles únicamente los accesorios para tubería de acero comercial o soldado, esto se ilustra en la figura 8.

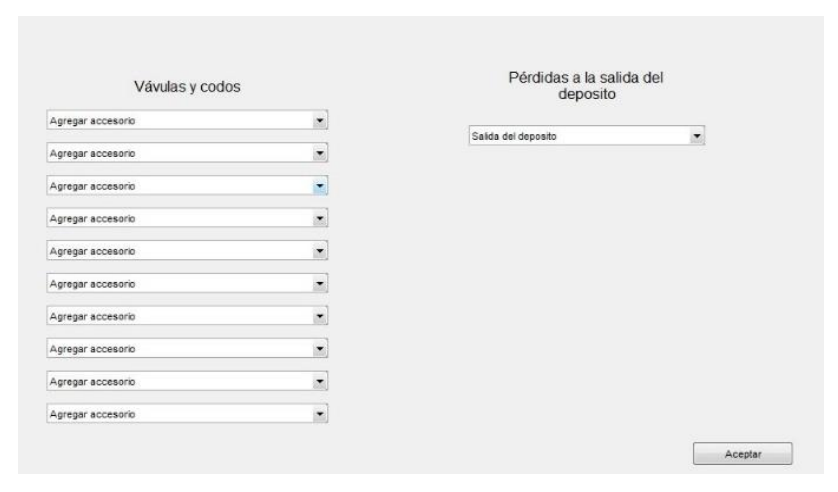

Figura 8 Ventana de accesorios Fuente: Elaboración Propia
Esta ventana permite elegir entre 10 tipos de accesorios, entre ellos: válvulas de globo, de ángulo, de compuerta, de verificación tipo bola y giratorio, de mariposa, válvula de pie tipo vástago y bisagra, codos roscados y estándar a $45^{\circ}$ y $90^{\circ}$, vueltas cerradas en retorno y $\mathrm{Te}$ estándar tanto en flujo directo como con flujo en el ramal.

\section{Comparativa entre método analítico tradicional y solución con PAST (con accesorios)}

Para calcular el tamaño de la tubería nueva y limpia de cédula 40 que conducirá 0.50 pies $3 / \mathrm{seg}$ de agua a $60^{\circ} \mathrm{F}$, incluye una válvula de mariposa abierta por completo y dos codos de radio largo; restrinja la caída de presión a 2.00psi en una longitud de 100 pies de tubería horizontal. Considerando que para el análisis de sistemas de tubería de clase III con accesorios se toma como base el diámetro nominal del análisis de sistemas de tuberías de clase III sin accesorios (Mott, Mecánica de fluidos $6^{\mathrm{a}}$ edición, 2006; Mataix, Mecánica de fluidos y máquinas hidráulicas, 2009; Streeter, 1999). En primer lugar, se calcula la pérdida de energía limitante, observando que la diferencia de elevación es igual a cero y el cambio de velocidades es tan pequeño que se considera despreciable. Partiendo de estas simplificaciones teóricas se tiene lo siguiente:

$$
\begin{aligned}
& \frac{P_{1}}{\gamma}+Z_{1}+\frac{v_{1}^{2}}{2 g}-h_{L}=\frac{P_{2}}{\gamma}+Z_{2}+\frac{v_{2}^{2}}{2 g} \\
& h_{L}=\frac{P_{1}-P_{2}}{\gamma} \\
& h_{L}=\frac{2 l i b / \text { pulg }^{2}}{62.4 \mathrm{lib}_{\text {pie }}}\left[\frac{12 \mathrm{pulg}}{1 \mathrm{pie}}\right]^{2} \\
& h_{L}=4.62 \text { pies }
\end{aligned}
$$

La rugosidad relativa del material, viscosidad cinemática y peso específico del agua a $60^{\circ} \mathrm{F}$ se puede consultar en el apéndice A del libro Mecánica de Fluidos (Mott, Mecánica de fluidos $6^{a}$ edición, 2006). Enseguida se introduce el valor de hL en la expresión del diámetro para encontrar el valor del diámetro calculado

$$
\begin{aligned}
& D=(0.66)\left[\epsilon^{1.25}\left[\frac{L Q^{2}}{g h_{L}}\right]^{4.75}+\right. \\
& \left.v Q^{9.4}\left[\frac{L}{g h_{L}}\right]^{5.2}\right]^{0.04}
\end{aligned}
$$




$$
\begin{aligned}
& D= \\
& (0.66)\left[\left(1.5 \times 10^{-4}\right)^{1.25}\left[\frac{(100)(0.5)^{2}}{(32.2)(4.62)}\right]^{4.75}+\right. \\
& \left.\left(1.21 \times 10^{-5}\right)(0.5)^{9.4}\left[\frac{100}{(32.2)(4.62)}\right]^{5.2}\right]^{0.04} \\
& D=0.3089 \text { pies }
\end{aligned}
$$

Se puede verificar en las tablas de acero comercial o soldado para tuberías de acero cédula 40, apéndice $\mathrm{F}$ del libro Mecánica de fluidos (Mott, Mecánica de fluidos $6^{\mathrm{a}}$ edición, 2006). El diámetro que se estará utilizando será el diámetro calculado sin accesorios de $\mathrm{D}=$ 0.3355 pies. Para simular una caída de presión deseada de 2.0psi. Después se examinará el valor resultante de la presión en el punto 2 para ver si es mayor o igual a 0psi, como se muestra en la ecuación (5)

$$
\begin{aligned}
& P_{2}=P_{1}+\Delta P \\
& P_{2}=0 P S I
\end{aligned}
$$

Para determinar el área se emplea la ecuación (6), para el caudal la ecuación (7) y para la velocidad la ecuación (8)

$$
\begin{aligned}
A & =\frac{\pi D^{2}}{4} \\
A & =\frac{\pi\left(0.335 \mathrm{pies}^{2}\right.}{4}=0.0884 \\
Q & =A v \\
v & =\frac{Q}{A} \\
v & =\frac{0.5 \mathrm{pies}^{3} / \mathrm{seg}}{0.0884 \mathrm{pies}^{2}}=5.55 \mathrm{pies} / \mathrm{seg}
\end{aligned}
$$

Después se determina el Número de Reynolds y la Rugosidad Relativa usando las ecuaciones (9) y (10)

$$
\begin{aligned}
& N_{R}=\frac{v D}{v} \\
& N_{R}=\frac{(5.55 \mathrm{pies} / \mathrm{seg})(0.3355 \mathrm{pies})}{1.21 \times 10^{-5} \mathrm{pies}^{2} / \mathrm{seg}} \\
& \quad=153886.3636 \\
& \quad \text { Rugosidad Relativa }=\frac{\varepsilon}{D} \quad(10) \\
& \text { Rugosidad Relativa }=\frac{1.50 \times 10^{-4} \text { pies }}{0.3355 \text { pies }}= \\
& 0.0000447093
\end{aligned}
$$

Después se determina el factor de fricción a través de la ecuación (11)

$$
f=\frac{0.25}{\left[\log _{10}\left(\frac{\varepsilon}{\frac{\varepsilon}{3.7}}+\frac{5.74}{N_{R} 0.9}\right)\right]^{2}}
$$

$$
f=\frac{0.25}{\left[\log _{10}\left(\frac{\frac{1.5 \times 10^{-4} \text { pies }}{0.3355 \text { pies }}}{3.7}+\frac{5.74}{(153886.3636)^{0.9}}\right)\right]^{2}}=
$$

Determinando las pérdidas de energía a través de la ecuación (12)

$$
\begin{gathered}
h_{L}=h_{\text {LTUBERÍA }}+2 h_{\text {LCODOS }}+ \\
h_{\text {LVÁLVULA MARIPOSA }} \text { (12) }
\end{gathered}
$$

$$
\begin{aligned}
& h_{L}=f_{T U B}\left(\frac{L_{T U B}}{D}\right)\left(\frac{v^{2}}{2 g}\right)+2 f_{C O D}\left(\frac{L_{e}}{D}\right)\left(\frac{v^{2}}{2 g}\right)+ \\
& f_{V A L}\left(\frac{L_{e}}{D}\right)\left(\frac{v^{2}}{2 g}\right)
\end{aligned}
$$

Tomando los valores correspondientes y resolviendo se obtiene la pérdida energética

$$
h_{L}=3.4994 \text { pies }
$$

Despejando la presión para el punto 2 de la ecuación general de la energía con el valor de las pérdidas de energía calculadas previamente, esto de acuerdo a lo mostrado en la ecuación (13)

$p_{\text {2real }}=p_{1}-\gamma\left(z_{1}-z_{2}+\frac{\left(v_{1}\right)^{2}-\left(v_{2}\right)^{2}}{2 g}-h_{L}\right)$

Evaluando la expresión con los datos obtenidos de los apéndices del libro de Mecánica de fluidos (Mott, Mecánica de fluidos $6^{\mathrm{a}}$ edición, 2006) se obtiene:

$$
p_{\text {2real }}=0.48359 \text { psi }
$$

Se acostumbra usar etapas que se pueden identificar con letras como en el ejemplo:

Este resultado muestra que la presión en el punto 2, en el extremo del sistema es de 0.4835 psi. Siendo el diseño satisfactorio. Las pérdidas provocadas por los codos y válvulas son en verdad pérdidas menores. La dimensión nominal de tubería comercial que cubre estas especificaciones corresponde a la tubería de acero de 4 pulgadas cédula 40 , con diámetro interno de $\mathrm{D}=0.3355$ pies. Si se realiza el cálculo usando el software PAST, una vez que se elige el sistema de unidades se debe introducir cada parámetro en el campo correspondiente. Se ingresa la diferencia de presiones, el cambio en la elevación y la temperatura del fluido, como se ilustra en la figura 9. 


\begin{tabular}{|c|c|c|}
\hline Longitud: & 100 & pies \\
\hline Presion 1: & 2 & Psi \\
\hline Presion 2: & o & Psi \\
\hline Altura 1: & o & pies \\
\hline Altura 2: & o & pies \\
\hline $\begin{array}{c}\text { Temperatura } \\
:\end{array}$ & 60 & $\circ \mathrm{F}$ \\
\hline
\end{tabular}

Figura 9 Parámetros de diseño para la tubería Fuente: Elaboración Propia

Una vez que se elige el material de la tubería, el tipo de cédula y se habilitan los accesorios; aparecerá la ventana que permite seleccionar los accesorios que se indican para cada caso, así como el tipo de salida del depósito a la tubería para incluir el efecto de esa pérdida energética, esta ventana se muestra en la figura 10

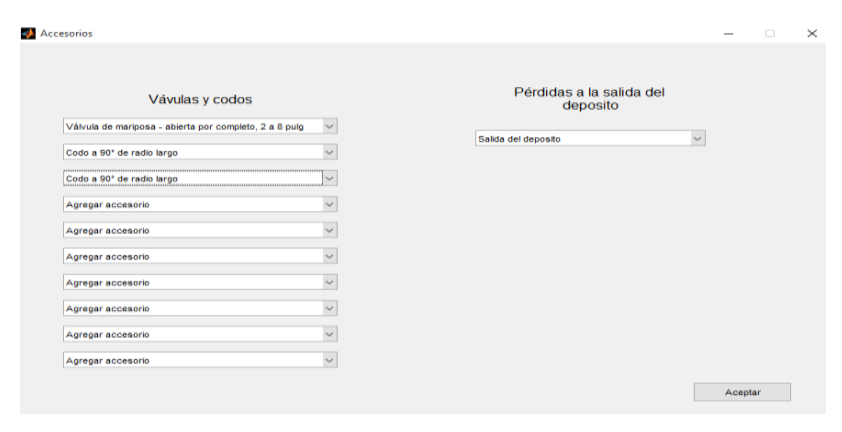

Figura 10 Ventana de accesorios Fuente: Elaboración Propia

Una vez que todos los parámetros se han ingresado, se seleccionaron los accesorios y el tipo de salida del depósito. En la figura 11 se presenta el botón de calcular y el resultado obtenido.

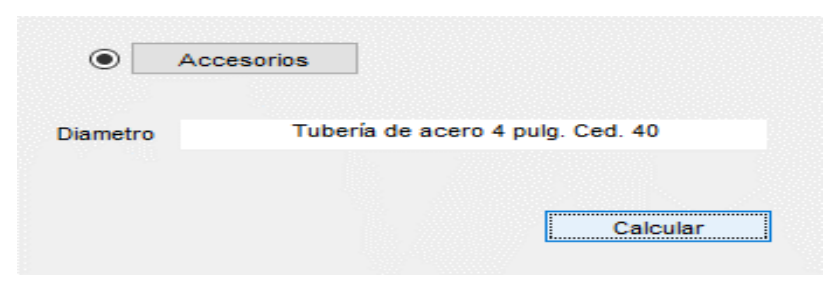

Figura 11 Botón de calcular y tubería resultante Fuente: Elaboración Propia

Se puede observar que el resultado en esencia es el mismo, pero obtenido mediante técnicas de manipulación de grandes volúmenes de datos de forma rápida y confiable como lo indica el Big Data.

\section{Resultados}

Para evaluar la funcionalidad de la aplicación PAST (Programa de Análisis de Sistemas de Tubería, nombre asignado a la aplicación), se consideraron diferentes ejercicios tomados del libro Mecánica de Fluidos sexta edición de Robert L. Mott, del capítulo 11 Sistemas de tuberías en serie para los sistemas de clase III; estos ejercicios fueron resueltos por alumnos de la carrera de Ingeniería Electromecánica del Instituto Tecnológico Superior de Irapuato, estos estudiantes estaban cursando o habían aprobado la materia de Mecánica de fluidos. Con esta prueba se obtuvo el tiempo que demoraban en resolver los problemas, después se resolvieron los ejercicios usando la aplicación. Los resultados obtenidos en esta etapa se pueden apreciar en la figura 12 .

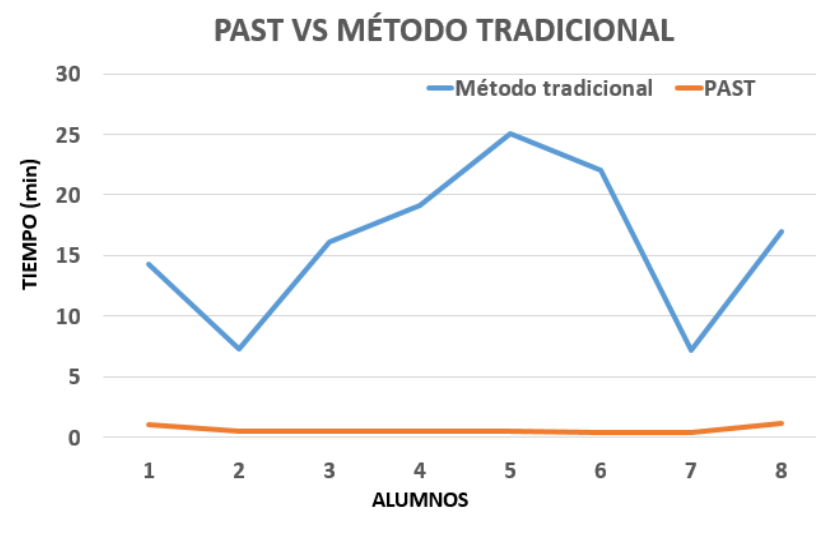

Figura 12 Programa de Análisis de Sistemas de Tuberías de Clase III contra el método analítico tradicional Fuente: Elaboración Propia

Después de obtener los tiempos de solución entre los métodos usados, se determinaron los promedios que se muestran en la tabla 1.

\begin{tabular}{|l|l|}
\hline \multicolumn{1}{|c|}{ Método empleado } & Promedio (min) \\
\hline Metodo tradicional & $16: 14$ \\
\hline Programa computacional & $00: 49$ \\
\hline
\end{tabular}

Tabla 5 Promedios de tiempos de solución Fuente: Elaboración Propia

Como se puede observar, el tiempo de solución se reduce considerablemente para los sistemas de tuberías de clase III, usando el programa computacional PAST, cabe aclarar que los problemas que se tomaron como referencia fueron los primeros del capítulo antes mencionado. 
Si se hubieran propuesto problemas con accesorios el tiempo requerido para el cálculo habría incrementado drásticamente debido al análisis complejo que se requiere para su solución. El algoritmo de solución del programa PAST es flexible y reconfigurable, permitiendo de esta manera soluciones obtenidas de grandes volúmenes de datos a través del empleo de técnicas de Big Data; esto sin comprometer la confiabilidad de los resultados entre un enorme volumen de datos variables que se seleccionan con velocidad, pero manteniendo la veracidad y el valor de los mismos.

El programa PAST ofrece la posibilidad de seleccionar entre un catálogo para materiales de tubería, incluir entre diferentes accesorios, advertencias cuando se presentan inconsistencias o campos vacíos; además de ser intuitivo y preciso. Una de las grandes ventajas es que el software obtiene prácticamente cualquier propiedad del agua a cualquier temperatura, además de incluir las tablas de los diferentes materiales para tuberías disponibles comercialmente. Para esta versión se considera únicamente el agua como el fluido de trabajo, en versiones posteriores se incluirán diferentes fluidos. Para esta aplicación se incluye un número de accesorios restringido. Otra desventaja es la compatibilidad con el sistema operativo ya sea de 32 o 64 bits.

\section{Conclusiones}

El programa PAST, desarrollado como una interfaz gráfica en el lenguaje de programación de Matlab®, ha demostrado ser una herramienta confiable y precisa que emplea técnicas de Big Data para la manipulación de grandes volúmenes de información con rapidez y confiabilidad. Ingresando los parámetros correctos durante la caracterización del sistema de tuberías de clase III, el programa determina el tamaño de tubería comercial requerido para las condiciones de operación además de reducir los tiempos de cálculo sin comprometer la confiabilidad del sistema. El PAST se concibe como una alternativa a los programas comerciales para el cálculo de sistemas residenciales, sistemas de riego, aplicaciones académicas e industriales; además de que el costo de la licencia de Matlab ${ }^{\circledR}$ es más bajo que el de aplicaciones comunes.

\section{Observaciones}

Para el caso de materiales de tubería no listados entre los disponibles del catálogo de la aplicación se cuenta con la opción de "otros" en los materiales de las tuberías; esto permite ingresar el valor de la rugosidad a través de una ventana como la que se muestra en la figura 13

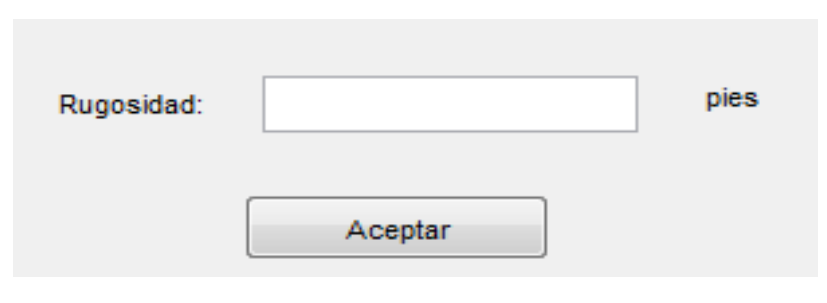

Figura 13 Ventana emergente para ingresar la rugosidad de otros materiales

Fuente: Elaboración Propia

\section{Recomendaciones}

El algoritmo diseñado para esta aplicación es flexible y fácilmente reconfigurable para usar las técnicas del Big Data y se puede emplear para solucionar problemas de diferentes campos ingenieriles tan diversos como sistemas hidráulicos, refrigeración, lubricación, selección de conductores eléctricos y en esencia cualquier problema que involucre la obtención de datos de tablas y catálogos.

\section{Referencias}

[1]. Cervantes, O., \& Báez, D. (2012). MATLAB con aplicaciones a la ingeniería, Física y Finanzas. CDMX, México: Alfaomega. K. M. Retzlaff and W. A. Ruegger, "Steam Turbines for Ultrasupercritical Power Plants", GE Power Systems, Schenectady, NY, GER3945a, 1-18, 1996.

[2]. M Jiménez-Medina, O. (2015). Fórmulas generales para los coeficientes de Chèzy y de Manning. Tecnología y ciencias del agua (ScieELO), 33-38.

[3]. Mataix, C. (2009). Mecánica de fluidos y máquinas hidráulicas. Alfaomega.

[4]. Mott, R. L. (2006). Mecánica de fluidos $6^{\mathrm{a}}$ edición. México: Prentice-Hall.

[5]. Streeter, V. (1999). Mecánica de fluidos. México: MCGRAW-HILL 
[6]. Bentley. (2018). WATERCAD.

Obtenido de

https://www.bentley.com/es/products/product-

line/hydraulics-and-hydrology-

software/watercad

[7]. Charter-plastics. (2018). charter plastic. Obtenido de http://www.charterplastics.com/

[8]. Copper-Alliance. (2018). Copper Development Alliance. Obtenido de https://www.copper.org/

[9]. Dupra. (2015). Ductile Iron Pipe Research Association. Obtenido de http://www.dipra.org/

[10]. Epanet. (17 de enero de 2017). Obtenido de https://www.epa.gov/water-research/epanet

[11]. Herco, R. (2018). Ryan Herco Flow Solutions. Obtenido de https://www.rhfs.com/

[12]. Nacobre. (2016). nacobre. Obtenido de http://www.nacobre.com.mx/download/ayudas/ nuevos/catalogo_nacobre_2016-2017.pdf

[13]. Oracle. (2013). Oracle. Obtenido de https://www.oracle.com/mx/bigdata/guide/what-is-big-data.html

[14]. PPI. (2018). Plastic Pipe Institute. Obtenido de https://www.plasticpipe.org/

[15]. Sicular, S. (27 de marzo de 2013). Forbes. Obtenido de https://www.forbes.com/sites/gartnergroup/201 3/03/27/gartners-big-data-definition-consistsof-three-parts-not-to-be-confused-with-threevs/\#5f2ad27542f6

[16]. TubeNet. (2018). Tubenet. Obtenido de http://www.tubenet.org.uk/tubes.shtml

[17]. Vidrasa. (2017). VIDRASA. Obtenido de

http://www.vidrasa.com/esp/productos/duratan/ duratan.html 